\title{
Upper-Extremity and Mobility Subdomains From the Patient- Reported Outcomes Measurement Information System (PROMIS) Adult Physical Functioning Item Bank
}

\author{
Ron D. Hays, PhD ${ }^{a, b}$, Karen L. Spritzer, BS ${ }^{a}$, Dagmar Amtmann, $\mathbf{P h D}^{c}$, Jin-Shei Lai, $\mathbf{P h D}^{\mathrm{d}}$, \\ Esi Morgan DeWitt, MD ${ }^{\mathrm{e}}$, Nan Rothrock, PhD $^{\mathrm{d}}$, Darren A. DeWalt, MD ${ }^{f}$, William T. Riley, \\ PhDg, James F. Fries, MD $^{h}$, and Eswar Krishnan, MD $^{h}$ \\ aUCLA Division of General Internal Medicine and Health Services Research, Department of \\ Medicine, Los Angeles, CA \\ ${ }^{b}$ RAND, Santa Monica, CA \\ 'Department of Rehabilitation Medicine, University of Washington, Seattle, WA \\ dDepartment of Medical Social Sciences, Northwestern University Feinberg School of Medicine, \\ Chicago, IL \\ eDepartment of Pediatrics, Cincinnati Children's Hospital Medical Center, Cincinnati, $\mathrm{OH}$ \\ fDivision of General Medicine and Clinical Epidemiology, Cecil G. Sheps Center for Health \\ Services Research, University of North Carolina at Chapel Hill \\ 9Science of Research and Technology Branch, Division of Cancer Control and Population \\ Sciences, National Cancer Institute, Bethesda, MD \\ hDepartment of Medicine, Stanford University School of Medicine, Stanford, CA
}

\section{Abstract}

Objective-To create upper-extremity and mobility subdomain scores from the Patient-Reported Outcomes Measurement Information System (PROMIS) physical functioning adult item bank.

\begin{abstract}
Design-Expert reviews were used to identify upper-extremity and mobility items from the PROMIS item bank. Psychometric analyses were conducted to assess empirical support for scoring upper-extremity and mobility subdomains.
\end{abstract}

Setting—Data were collected from the U.S. general population and multiple disease groups via self-administered surveys.

Participants-The sample ( $\mathrm{N}=21,773)$ included 21,133 English-speaking adults who participated in the PROMIS wave 1 data collection and 640 Spanish-speaking Latino adults recruited separately.

Interventions-Not applicable.

Main Outcome Measures-We used English- and Spanish-language data and existing PROMIS item parameters for the physical functioning item bank to estimate upper-extremity and

\footnotetext{
(C) 2013 by the American Congress of Rehabilitation Medicine

Corresponding author Ron D. Hays, PhD, UCLA Division of General Internal Medicine and Health Services Research, Department of Medicine, 911 Broxton Ave, Los Angeles, CA 90095. drhays@ucla.edu..

No commercial party having a direct financial interest in the results of the research supporting this article has or will confer a benefit on the authors or on any organization with which the authors are associated.
} 
mobility scores. In addition, we fit graded response models to calibrate the upper-extremity items and mobility items separately, compare separate to combined calibrations, and produce subdomain scores.

Results-After eliminating items because of local dependency, 16 items remained to assess upper extremity and 17 items to assess mobility. The estimated correlation between upper extremity and mobility was .59 using existing PROMIS physical functioning item parameters ( $r=$. 60 using parameters calibrated separately for upper-extremity and mobility items).

Conclusions-Upper-extremity and mobility subdomains shared about $35 \%$ of the variance in common, and produced comparable scores whether calibrated separately or together. The identification of the subset of items tapping these 2 aspects of physical functioning and scored using the existing PROMIS parameters provides the option of scoring these subdomains in addition to the overall physical functioning score.

\section{Keywords}

Lower extremity; Psychometrics; Rehabilitation; Upper extremity

Physical functioning is an especially important indicator of health and one of the strongest predictors of health care utilization and mortality. A physical functioning item bank was created for the Patient-Reported Outcomes Measurement Information System (PROMIS) project $^{1}$ that consists of 124 items assessing mobility (lower extremity), upper extremity, axial or central (neck and back) function, and complex activities that overlap with more than 1 domain (daily living activities). Despite the theoretical possibility of multiple subdomains, the PROMIS physical functioning items were found to be essentially unidimensional..$^{2-4}$ Analyses of other measures have yielded similar support for a single underlying physical functioning dimension. ${ }^{5}$

Although the PROMIS physical functioning bank is sufficiently unidimensional overall, it is important to consider anatomy-based or function-targeted aspects of physical functioning. For example, some aspects of physical functioning are more relevant to individuals with disabilities such as those using wheelchairs for mobility ${ }^{6}$ and may be more significant for certain clinical subgroups of the population such as patients with knee injuries, sciatica, amputation, or carpal tunnel syndrome. Targeted interventions can result in dramatic improvement in the anatomical or functional deficit but may not necessarily register in an overall measure of physical functioning. For example, multiple physical functioning subdomains were identified for persons with spinal cord injuries. ${ }^{7}$

There are a variety of upper-extremity ${ }^{8}$ and mobility ${ }^{9}$ measures, 2 subdomains of physical functioning. DeWitt et $\mathrm{al}^{10}$ found that the PROMIS pediatric physical functioning items were best represented by upper-extremity and mobility subdomains. Another study ${ }^{11}$ administered the PROMIS physical functioning item bank to a sample of 865 adult patients being seen for musculoskeletal problems and found that the bank was sufficiently unidimensional, but there was a nontrivial amount of variance representing the distinction between upper extremity and mobility. In addition, several items displayed differential item functioning between patients with upper-extremity $(n=365)$ and mobility problems $(n=500)$. Hung et a ${ }^{12}$ selected 79 items from the PROMIS physical functioning bank and referred to this as a "lower-extremity" bank based on administering the PROMIS items to a sample of 382 orthopedic patients with lower-extremity complaints. But almost half the items (37 items) in this subbank assess aspects of physical functioning other than mobility (ie, instrumental aspects of daily living, axial and central activities). In addition, the relation of scores estimated from this subset of PROMIS physical functioning items with upper extremity is unknown. 
The goal of the present study was to identify and aggregate subsets of items targeting upper extremity and mobility within the existing PROMIS physical functioning item bank and to evaluate the evidence for creating separate subdomain scores.

\section{Methods \\ PROMIS items}

The PROMIS version 1 English-language adult physical functioning bank with 124 items assesses one's ability to carry out activities that require physical actions, ranging from selfcare to more complex activities that require a combination of skills, often within a social context. The majority of the items (114 of the 124 items) were translated into Spanish using a universal approach for translations and cultural adaption of instruments. ${ }^{13}$ (The 10 items not translated were those with the most restricted distribution of responses in the English sample such as "Are you able to open previously opened jars?")

\section{Administration of items}

A randomly selected subset of English-language respondents was administered blocks of 7 items from each of 14 PROMIS domains. ${ }^{1}$ Therefore, these respondents completed only 7 randomly selected physical functioning items. Another randomly selected subset of respondents was administered 2 sets of 56 physical functioning items (112 items in total), and another was administered 1 set of 56 physical functioning items. Because of the mix of block and full bank administration and large sample size, there was sufficient sample for each item pair to evaluate correlations among items and evaluate potential subdomains.

Subsequently, the 114 items noted above were administered to Spanish-speaking Latinos.

\section{Participants}

From July 2006 to March 2007, data were collected from English-language adults from the U.S. general population and multiple disease groups $(n=21,133)$. Of these, 1,532 were recruited from primary research sites associated with PROMIS network sites. The majority of the data was collected by YouGovPolimetrix, a polling firm based in Palo Alto, CA. This firm uses a sample-matching procedure to select a representative sample of the population. The respondents had similar demographic characteristics as the U.S. census, except that the online panel tended to have more educated individuals. ${ }^{14}$ The Spanish sample included 640 adult Spanish-speaking Latinos in the Toluna online panel, an independent survey technology provider. All 640 respondents answered all the 114 physical functioning items administered to them. Sample sizes for analyses vary because different respondents were randomized to varying combinations of items from the physical functioning item pool in PROMIS.

\section{Identifying and categorizing upper-extremity and mobility items}

We began by sorting the PROMIS physical functioning items into upper-extremity, mobility, and "both" categories. "Both" was defined as an item that appeared to require both upper-extremity and mobility capabilities to perform and, therefore, would not be used to create upper-extremity or mobility subdomain scores. The PROMIS investigators and nonPROMIS experts reviewed the initial classification of items, and the list was revised accordingly. For example, 1 item originally classified as mobility (Physical Functioning-A [PFA]12: "Are you able to push open a heavy door?") was deemed to require both upperextremity and trunk involvement. This item was reclassified into the "both" category. An item originally classified as upper extremity (Physical Functioning-B [PFB]43: "Does your health now limit you in taking care of your personal needs [dress, comb hair, toilet, eat, bathe]?") was also reclassified into the "both" category because it was deemed to require 
elements of trunk stability as well. After review, we identified 27 upper-extremity items and 35 mobility items (the remaining 52 items were included in the "both" category).

\section{Analysis plan}

We fit categorical confirmatory factor analytic models to the upper-extremity items and mobility items using Mplus Version $6{ }^{\text {a }}$ Items were deleted when item pairs had residual correlations of .20 or higher. We then estimated upper-extremity and mobility subdomain scores using existing PROMIS item parameters (slope and threshold) where both upperextremity and mobility items were calibrated together using Samejima's graded response model. This model yields 1 slope or discrimination parameter and (n-1) threshold parameters for polytomous items with $\mathrm{n}$ response options. The slope parameter gives information regarding the discrimination of the item between adjoining trait levels. Higher values indicate that items are better able to discriminate between adjacent categories of trait level. The threshold parameter represents the point along the latent trait at which a respondent has a $50 \%$ chance of responding in that category or higher. We also calibrated new item parameters separately for the upper-extremity items and the mobility items fitting the same Samejima's graded response model as the current PROMIS physical functioning item bank. MULTILOG software ${ }^{b}$ was used to estimate the item parameters.

We analyzed the English- and Spanish-language data together in this study to maximize the precision of statistical estimates. An analysis of differential item functioning for the physical functioning items by language was previously reported, and the results supported this approach. ${ }^{15}$

\section{Results}

A 2-factor categorical factor analytic model for the 27 upper-extremity and 35 mobility items and 20 correlated uniqueness terms (ie, residual correlations among item pairs) was rejectable statistically $\left[\mathrm{X}^{2}(\mathrm{n}=16,357 ; d f=1,808)=41,038.55\right]$, but fit the data reasonably well (Confirmatory Fit Index [CFI]=.940; Root Mean Square Error of Approximation [RMSEA] $=.036$ ). One of the mobility items (Physical Functioning-C [PFC]33, Are you able to run 10 miles?) had large residual correlations of .95 with PFC7 (Are you able to run 5 miles), .91 with PFA39 (Are you able to run at a fast pace for 2 miles), .58 with PFA19 (Are you able to run or jog for 2 miles), .51 with PFC32 (Are you able to climb up 5 flights of stairs), and .43 with PFC13 (Are you able to run 100 yards?) Hence, we eliminated this item (PFC33) from the mobility item subset, resulting in 27 upper-extremity items and 34 mobility items. We then iteratively deleted items until all residual correlations within the upper-extremity and mobility subdomains were $<.20$. This resulted in 16 upper-extremity and 17 mobility items that satisfied the local dependency assumption (see tables 1 and 2).

A 2-factor categorical factor analysis model for these items was rejectable statistically $\left[\mathrm{X}^{2}(\mathrm{n}=16,346 ; d f=494)=10,666.88\right]$, but fit the data well $(\mathrm{CFI}=.971$; RMSEA=.035). A 1factor categorical factor analysis model for these items fit the data significantly less well $\left[\mathrm{X}^{2}(\mathrm{n}=16,346 ; d f=495)=11,220.62 ; \mathrm{CFI}=.970 ; \mathrm{RMSEA}=.036\right]$ than did the 2-factor model. One-factor models were also estimated separately for the 16 upper-extremity items (CFI=. 993 and RMSEA=.032) and the 17 mobility items (CFI=.996 and RMSEA=.023).

Standardized factor loadings for the upper-extremity items are provided in table 1; the loadings for the mobility items are given in table 2 . All loadings were statistically significant and large.

The threshold estimates in tables 1 and 2 provide information about the difficulties of the items. The items are targeted at lower levels of physical functioning. Even people with relatively low levels of physical functioning ( -0.93 to $-1.95 \mathrm{Z}$ score for upper extremity, 
-0.11 to -1.14 for mobility) have a $50 \%$ probability of picking the most extreme positive response on the items. The skewness and kurtosis for the estimated upper-extremity (mobility) scores in the sample were $-1.70(-0.88)$ and $2.19(-0.07)$, with $<1 \%(<1 \%)$ scoring at the floor (lowest possible score) and 54\% (27\%) scoring at the ceiling (highest possible score).

The PROMIS physical functioning upper-extremity and mobility subdomains correlated .59 with one another (table 3). In addition, the upper-extremity subdomain correlated .52 with the PROMIS global physical health scale, .32 with the PROMIS global mental health scale, and .64 with the overall PROMIS physical functioning scale. The mobility subdomain correlated .71 with the PROMIS global physical health scale, .38 with the PROMIS global mental health scale, and .87 with the overall PROMIS physical functioning scale. (Appendix table 1 shows the same correlations based on calibrations of the upper-extremity and mobility items separately within each domain.)

\section{Discussion}

One goal of PROMIS is to improve precision and the validity of health outcome measures. Although the PROMIS physical functioning item bank has been found to be essentially unidimensional in empirical analyses, it was constructed to represent subdomains of physical functioning. This reflects the fact that those with chronic illnesses often have problems with multiple aspects of physical functioning. However, some researchers and clinicians have expressed interest in being able to separately evaluate upper extremity and mobility for certain subgroups of the population (eg, orthopedic patients). This study identified a subset of 16 upper-extremity and 17 mobility items from the PROMIS physical functioning item bank based on item content that satisfied the assumptions of item response theory. These items were used to create upper-extremity and mobility subdomain scores using the existing PROMIS physical functioning item parameters.

The upper-extremity and mobility subdomains share approximately $35 \%$ of the variance in common ( $r=.59)$. The mobility subdomain was highly correlated with the overall PROMIS physical functioning score $(r=.87)$, while the upper-extremity subdomain correlated less strongly $(r=.64)$. Hence, the upper-extremity subdomain provides more distinct information than mobility compared with the overall PROMIS physical functioning domain. As noted above, mobility was also more highly correlated than upper extremity with the PROMIS global physical health ( $r=.71 \mathrm{vs} .52)$ and PROMIS global mental health ( $r=.38 \mathrm{vs} .32)$ scales. Therefore, the upper extremity may yield different results and be more sensitive to change than the overall physical functioning domain for some conditions such as carpal tunnel syndrome.

\section{Study limitations}

We note that other approaches could be used to select subsets of upper-extremity and mobility subdomain items. For example, a Rasch model could be used to evaluate the fit of the items initially selected. In addition, future work applying multidimensional item response theory models could be used to improve the precision of the estimated subdomain scores. ${ }^{16,17}$

\section{Conclusions}

Given the minimal differences between calibrating upper-extremity and mobility items separately and using the original PROMIS item parameters, we recommend that the published PROMIS item parameters be used to score the upper-extremity and mobility subdomains (see http://www.assessmentcenter.net) using "response pattern scoring." Raw 
score to T-score conversion tables are also provided on the Web site at https:// www.assessmentcenter.net/documents/PROMIS\%20Physical\%20Function\%20Scoring \%20Manual.pdf. On the basis of correlations from the PROMIS data collection reported here, one would expect the upper-extremity subdomain to have a higher likelihood than mobility of yielding unique information. However, the estimated correlations may vary in different subgroups. Additional research in targeted subgroups is needed to obtain additional evidence about the associations among upper extremity and mobility.

Of the 16 upper-extremity and 17 mobility items identified from the PROMIS adult physical function bank, there are 8 upper-extremity and 6 mobility items from the PROMIS pediatric banks that assess similar content (see table 4). This overlap provides the basis for a future linking study that may allow researchers to follow patients from childhood through adulthood on upper-extremity and mobility physical functioning.

\section{Suppliers}

a. Mplus Version 6; Muthén \& Muthén, 3463 Stoner Ave, Los Angeles, CA 90066.

b. MULTILOG Version 7.0.3; Scientific Software International, Inc, P.O. Box 4728, Skokie, IL 60076-4728.

\section{Acknowledgments}

The Patient-Reported Outcomes Measurement Information System (PROMIS) is a National Institutes of Health (NIH) Roadmap initiative to develop a computerized system measuring patient-reported outcomes in respondents with a wide range of chronic diseases and demographic characteristics. NIH Science Officers on this project have included Deborah Ader, PhD, Vanessa Ameen, MD, Susan Czajkowski, PhD, Basil Eldadah, MD, PhD, Lawrence Fine, MD, DrPH, Lawrence Fox, MD, PhD, Lynne Haverkos, MD, MPH, Thomas Hilton, PhD, Laura Lee Johnson, $\mathrm{PhD}$, Michael Kozak, PhD, Peter Lyster, PhD, Donald Mattison, MD, Claudia Moy, PhD, Louis Quatrano, PhD, Bryce Reeve, PhD, William Riley, PhD, Ashley Wilder Smith, PhD, MPH, Susana Serrate-Sztein, MD, Ellen Werner, $\mathrm{PhD}$, and James Witter, MD, PhD. This manuscript was reviewed by PROMIS reviewers before submission for external peer review. See the Web site at www.nihpromis.org for additional information on the PROMIS initiative. We thank 2 prosthetists, Danny Abrahamson and Brian Hafner, for initially sorting the items into upper-extremity, mobility, and both bins. The article's contents are solely the responsibility of the authors and do not necessarily represent the official views of the NIH.

PROMIS II was funded by cooperative agreements with a Statistical Center (Northwestern University, PI: David Cella, PhD, 1U54AR057951), a Technology Center (Northwestern University, PI: Richard C. Gershon, PhD, 1U54AR057943), a Network Center (American Institutes for Research, PI: Susan [San] D. Keller, PhD, 1U54AR057926), and 13 primary research sites that may include more than 1 institution (State University of New York, Stony Brook, PIs: Joan E. Broderick, PhD, and Arthur A. Stone, PhD, 1U01AR057948; University of Washington, Seattle, PIs: Heidi M. Crane, MD, MPH, Paul K. Crane, MD, MPH, and Donald L. Patrick, PhD, 1U01AR057954; University of Washington, Seattle, PIs: Dagmar Amtmann, PhD, and Karon Cook, PhD, 1U01AR052171; University of North Carolina, Chapel Hill, PI: Darren A. DeWalt, MD, MPH, 2U01AR052181; Children's Hospital of Philadelphia, PI: Christopher B. Forrest, MD, PhD, 1U01AR057956; Stanford University, PI: James F. Fries, MD, 2U01AR052158; Boston University, PIs: Stephen M. Haley, PhD, and David Scott Tulsky, $\mathrm{PhD}$ [University of Michigan, Ann Arbor], 1U01AR057929; University of California, Los Angeles, PIs: Dinesh Khanna, MD, and Brennan Spiegel, MD, MSHS, 1U01AR057936; University of Pittsburgh, PI: Paul A. Pilkonis, PhD, 2U01 AR052155; Georgetown University, PIs: Carol. M. Moinpour, PhD [Fred Hutchinson Cancer Research Center, Seattle], and Arnold L. Potosky, PhD, U01AR057971; Children's Hospital Medical Center, Cincinnati, PI: Esi M. Morgan DeWitt, MD, MSCE, 1U01AR057940; University of Maryland, Baltimore, PI: Lisa M. Shulman, MD, 1U01AR057967; and Duke University, PI: Kevin P. Weinfurt, PhD, 2U01AR052186). The study was also supported by UCLA/DREW Project EXPORT, NIMHD (grant no. 2P20MD000182) and in part by the AO Foundation. 


\section{Appendix}

\section{Table 1}

Correlations of upper-extremity and mobility subdomains with PROMIS global physical health, global mental health, and overall physical functioning domains (using separate calibrations of items within upper extremity and mobility)

\begin{tabular}{lcc}
\hline Domain & Upper Extremity & Mobility \\
\hline Mobility & $r=.60(\mathrm{n}=10,969)$ & \\
Global physical health & $r=.52(\mathrm{n}=12,655)$ & $r=.71(\mathrm{n}=14,660)$ \\
Global mental health & $r=.32(\mathrm{n}=12,655)$ & $r=.38(\mathrm{n}=14,660)$ \\
$\begin{array}{l}\text { Overall physical } \\
\text { functioning }\end{array}$ & $r=.64(\mathrm{n}=12,655)$ & $r=.86(\mathrm{n}=14,660)$ \\
\hline
\end{tabular}

\section{List of abbreviations}

$\begin{array}{ll}\text { CFI } & \text { Confirmatory Fit Index } \\ \text { PFA } & \text { Physical Functioning-A } \\ \text { PFB } & \text { Physical Functioning-B } \\ \text { PFC } & \text { Physical Functioning-C } \\ \text { PROMIS } & \text { Patient-Reported Outcomes Measurement Information System } \\ \text { RMSEA } & \text { Root Mean Square Error of Approximation }\end{array}$

\section{References}

1. Cella D, Riley W, Stone A, et al. The Patient-Reported Outcomes Measurement Information System (PROMIS) developed and tested its first wave of adult self-reported health outcome item banks: 2005-2008. J Clin Epidemiol. 2010; 63:1179-94. [PubMed: 20685078]

2. Bruce B, Fries JF, Ambrosini D, et al. Better assessment of physical function: item improvement is neglected but essential. Arthritis Res Ther. 2009; 1:R191. Available at: http://arthritis-research.com/ content/11/6/r191. [PubMed: 20015354]

3. Fries JF, Cella D, Rose M, Krishnan E, Bruce B. Progress in assessing physical function in arthritis: PROMIS short forms and computerized adaptive testing. J Rheumatol. 2009; 36:2061-6. [PubMed: 19738214]

4. Rose M, Bjorner JB, Becker J, et al. Evaluation of a preliminary physical function item bank supported the expected advantages of the Patient-Reported Outcomes Measurement Information System (PROMIS). J Clin Epidemiol. 2008; 61:17-33. [PubMed: 18083459]

5. Hays RD, Liu H, Spritzer K, Cella D. Item response theory analyses of physical functioning items in the Medical Outcomes Study. Med Care. 2007; 45:S32-8. [PubMed: 17443117]

6. Hays RD, Hahn H, Marshall G. Use of the SF-36 and other health-related quality of life measures to assess persons with disabilities. Arch Phys Med Rehabil. 2002; 83:S4-9. [PubMed: 12474166]

7. Tulsky DS, Jette AM, Kisala PA, et al. Spinal cord injury-functional index: item banks to measure physical functioning in individuals with spinal cord injury. Arch Phys Med Rehabil. 2012; 93:172232. [PubMed: 22609299]

8. Davis AM, Beaton DE, Hudak P, et al. Measuring disability of the upper extremity: a rationale supporting the use of a regional outcome measure. J Hand Ther. 1999; 12:269-74. [PubMed: 10622192]

9. Ferrucci L, Guralnik JM. Mobility in human aging: a multidisciplinary life span conceptual framework. Annu Rev Gerontol Geriatr. 2013; 33:171-92. 
10. DeWitt EM, Stucky BD, Thissen D, et al. Construction of the eight-item Patient-Reported Outcomes Measurement Information System pediatric physical function scales: built using item response theory. J Clin Epidemiol. 2011; 64:794-804. [PubMed: 21292444]

11. Hung M, Clegg DO, Greene T, Saltzman CL. Evaluation of the PROMIS physical function item bank in orthopaedic patients. J Orth Res. 2001; 29:947-53.

12. Hung M, Clegg DO, Greene T, Weir C, Saltzman CL. A lower extremity physical function computerized adaptive testing instrument for orthopaedic patients. Foot Ankle Int. 2012; 33:1-10. [PubMed: 22381229]

13. Eremenco SL, Cella D, Arnold BJ. A comprehensive method for the translation and cross-cultural validation of health status questionnaires. Eval Health Prof. 2005; 38:212-32. [PubMed: 15851774]

14. Liu HH, Cella D, Gershon R, et al. Representativeness of the PROMIS internet panel. J Clin Epidemiol. 2010; 63:1169-78. [PubMed: 20688473]

15. Paz SH, Spritzer KL, Morales LS, Hays RD. Evaluation of the Patient-Reported Outcomes Information System (PROMIS®) Spanish Physical Functioning Items. Qual Life Res. 2012 [epub ahead of print] PMID:23124505.

16. Haberman SJ, Sinharay S. Reporting of subscores using multidimensional item response theory. Psychometrika. 2010; 75:209-17.

17. Wang WC, Chen PH, Cheng YY. Improving measurement precision of test batteries using multidimensional item response models. Psychol Meth. 2004; 9:116-36. 
Table 1

Factor loadings for 16 upper-extremity items from category confirmatory factor analysis model $(\mathrm{n}=12,655)$

\begin{tabular}{|c|c|c|c|c|c|c|}
\hline \multirow[b]{2}{*}{ Loading } & \multirow[b]{2}{*}{ Standard Error } & \multicolumn{4}{|c|}{ Thresholds } & \multirow[b]{2}{*}{ Item } \\
\hline & & 1 & 2 & 3 & 4 & \\
\hline .926 & .007 & -4.05 & -3.40 & -2.71 & -1.76 & Open a new milk carton? (PFB30) \\
\hline .940 & .007 & -3.87 & -3.29 & -2.62 & -1.92 & Button your shirt? (PFA54) \\
\hline .955 & .006 & -3.87 & -3.01 & -2.39 & -1.69 & Pick up coins from a tabletop? (PFB21) \\
\hline .932 & .006 & -3.78 & -3.12 & -2.29 & -1.51 & Put on a pullover sweater? (PFB36) \\
\hline .938 & .006 & -3.77 & -3.08 & -2.47 & -1.85 & Open and close a zipper? (PFA35) \\
\hline .935 & .007 & -3.62 & -2.78 & -2.29 & -1.60 & Hold a plate full of food? (PFB22) \\
\hline .951 & .006 & -3.59 & -2.96 & -2.51 & -1.85 & Peel fruit? (PFA48) \\
\hline .921 & .007 & -3.52 & -2.91 & -2.38 & -1.75 & Remove something from your back pocket? (PFB33) \\
\hline .973 & .004 & -3.41 & -2.99 & -2.42 & -1.72 & Put on a shirt or blouse? (PFA44) \\
\hline .942 & .008 & -3.34 & -2.87 & -2.50 & -1.95 & Cut your food using eating utensils? (PFA20) \\
\hline .909 & .008 & -3.19 & -2.51 & -1.94 & -1.17 & Open a can with a hand can opener? (PFA28) \\
\hline .918 & .007 & -3.14 & -2.56 & -1.91 & -1.24 & Dress ...tying shoelaces and doing buttons? (PFA16) \\
\hline .923 & .006 & -3.03 & -2.51 & -1.94 & -1.39 & Dry your back with a towel? (PFA38) \\
\hline .906 & .008 & -2.92 & -2.47 & -1.94 & -1.34 & Use a hammer to pound a nail? (PFA18) \\
\hline .846 & .011 & -2.72 & -2.31 & -1.75 & -0.93 & Reach into a high cupboard? (PFA17) \\
\hline .845 & .010 & -2.60 & -2.06 & -1.51 & -0.93 & Pull heavy objects (10lb) toward yourself? (PFA29) \\
\hline
\end{tabular}

NOTE. The 4 thresholds for each item are shown in the middle columns. These values represent the estimated upper-extremity level on a $z$-score metric needed to have a 50\% probability of selecting a response higher (greater physical functioning) than the first, second, third, and fourth response categories, respectively. 
Table 2

Factor loadings for 17 mobility items from category confirmatory factor analysis model $(\mathrm{n}=14,660)$

\begin{tabular}{|c|c|c|c|c|c|c|}
\hline \multirow[b]{2}{*}{ Loading } & \multirow[b]{2}{*}{ Standard Error } & \multicolumn{4}{|c|}{ Thresholds } & \multirow[b]{2}{*}{ Item } \\
\hline & & 1 & 2 & 3 & 4 & \\
\hline .873 & .010 & -2.80 & -2.11 & -1.54 & -0.77 & Able to stand up from armless straight chair? (PFA15) \\
\hline .897 & .008 & -2.60 & -2.07 & -1.58 & -1.09 & Are you able to climb up 5 steps? (PFB10) \\
\hline .914 & .007 & -2.47 & -2.12 & -1.66 & -1.14 & Able to stand supported for 10min? (PFB32) \\
\hline .896 & .007 & -2.40 & -1.42 & -0.81 & -0.26 & Physical health problems limit activities? (PFA07) \\
\hline .919 & .006 & -2.31 & -1.63 & -1.05 & -0.57 & Climbing 1 flight of stairs? (PFC37) \\
\hline .865 & .009 & -2.28 & -1.91 & -1.55 & -0.98 & Are you able to stand up on tiptoes? (PFB40) \\
\hline .874 & .010 & -2.20 & -1.57 & -1.10 & -0.71 & Health limit you in walking 100y? (PFC20) \\
\hline .929 & .006 & -2.19 & -1.68 & -1.18 & -0.74 & Health limit you in going for a short walk? (PFB49) \\
\hline .884 & .008 & -2.13 & -1.55 & -1.05 & -0.31 & Get up off floor from lying on your back? (PFA31) \\
\hline .938 & .005 & -2.04 & -1.69 & -1.22 & -0.71 & Are you able to walk at a normal speed? (PFC38) \\
\hline .919 & .006 & -2.01 & -1.66 & -1.26 & -0.72 & Able to stand unsupported for 30min? (PFB42) \\
\hline .927 & .006 & -1.91 & -1.58 & -1.19 & -0.68 & Able to go for a walk of at least $15 \mathrm{~min}$ ? (PFA23) \\
\hline .923 & .005 & -1.89 & -1.48 & -1.05 & -0.40 & Able to go up and down stairs at normal pace? (PFA21) \\
\hline .915 & .006 & -1.81 & -1.15 & -0.56 & -0.11 & Climbing several flights of stairs? (PFC10) \\
\hline .895 & .006 & -1.75 & -1.35 & -0.92 & -0.34 & Are you able to stand for $1 \mathrm{~h}$ ? (PFA10) \\
\hline .907 & .006 & -1.65 & -1.28 & -0.88 & -0.37 & Are you able to jump up and down? (PFB09) \\
\hline .907 & .006 & -1.41 & -1.09 & -0.74 & -0.13 & Run short distance, such as to catch bus? (PFB24) \\
\hline
\end{tabular}

NOTE. The 4 thresholds for each item are shown in the middle columns. These values represent the estimated mobility level on a $z$-score metric needed to have a $50 \%$ probability of selecting a response higher (greater physical functioning) than the first, second, third, and fourth response categories, respectively. 


\section{Table 3}

Correlations of upper-extremity and mobility subdomains with PROMIS global physical health, global mental health, and overall physical functioning domains

\begin{tabular}{lcccc}
\hline Domain & Upper Extremity & Mobility & Global Physical Health & Global Mental Health \\
\hline Mobility & $r=.59(\mathrm{n}=10,969)$ & & & \\
Global physical health & $r=.52(\mathrm{n}=12,655)$ & $r=.71(\mathrm{n}=14,660)$ & & \\
Global mental health & $r=.32(\mathrm{n}=12,655)$ & $r=.38(\mathrm{n}=14,660)$ & $r=.62(\mathrm{n}=21,019)$ & $r=.43(\mathrm{n}=16,365)$ \\
Overall physical functioning & $r=.64(\mathrm{n}=12,655)$ & $r=.87(\mathrm{n}=14,660)$ & $r=.77(\mathrm{n}=16,365)$ & \\
\hline
\end{tabular}


Table 4

Similar PROMIS physical functioning items in pediatric and adult banks

\begin{tabular}{|c|c|}
\hline Adult Item & Pediatric Item \\
\hline Are you able to dress yourself, including tying shoelaces & I could put on my clothes by myself. \\
\hline and doing buttons? & I could tie shoelaces by myself. \\
\hline Are you able to open and close a zipper? & I could zip up my clothes. \\
\hline Are you able to dry your back with a towel? & I could dry my back with a towel. \\
\hline Are you able to turn a key in a lock? & I could use a key to unlock a door. \\
\hline Are you able to brush your teeth? & I could brush my teeth by myself. \\
\hline Are you able to button your shirt? & I could button my shirt or pants. \\
\hline Are you able to push open a door after turning the knob? & I could pull open heavy doors. \\
\hline Are you able to put on a pullover sweater? & I could pull a shirt on over my head by myself. \\
\hline Are you able to stand up from an armless straight chair? & I could get up from the floor. \\
\hline Are you able to run or jog for 2 miles? & I could run a mile. \\
\hline Are you able to step up and down curbs? & I could go up 1 step. \\
\hline Are you able to stand up on tiptoes? & I could stand up on my tiptoes. \\
\hline Are you able to walk a block on flat ground? & I could walk more than 1 block. \\
\hline Are you able to kneel on the floor? & I could get down on my knees without holding on to something. \\
\hline
\end{tabular}

